\title{
Entropy bounds and black hole remnants*
}

\author{
Jacob D. Bekenstein ${ }^{\dagger}$ \\ Department of Physics, University of California at Santa Barbara, Santa Barbara, CA 93106 \\ and \\ The Racah Institute of Physics, Hebrew University of Jerusalem, Givat Ram, Jerusalem 91904, Israel ${ }^{\ddagger}$
}

(Received)

\begin{abstract}
We rederive the universal bound on entropy with the help of black holes while allowing for Unruh-Wald buoyancy. We consider a box full of entropy lowered towards and then dropped into a Reissner-Nordström black hole in equilibrium with thermal radiation. We avoid the approximation that the buoyant pressure varies slowly across the box, and compute the buoyant force exactly. We find, in agreement with independent investigations, that the neutral point generically lies very near the horizon. A consequence is that in the generic case, the Unruh-Wald entropy restriction is neither necessary nor sufficient for enforcement of the generalized second law. Another consequence is that generically the buoyancy makes only a negligible contribution to the energy bookeeping, so that the original entropy bound is recovered if the generalized second law is assumed to hold. The number of particle species does not figure in the entropy bound, a point that has caused some perplexity. We demonstrate by explicit calculation that, for arbitrarily large number of particle species, the bound is indeed satisfied by cavity thermal radiation in the thermodynamic regime, provided vacuum energies are included. We also show directly that thermal radiation in a cavity in $D$ dimensional space also respects the bound regardless of the value of $D$. As an application of the bound we show that it strongly restricts the information capacity of the posited black hole remnants, so that they cannot serve to resolve the information paradox.
\end{abstract}

PACS numbers: 97.60.Lf, 95.30.Tg, 04.60.+n, 05.90.+m

\section{INTRODUCTION}

The generalized second law (GSL) of thermodynamics for black holes [1]2] states that when entropy flows into a black hole, the sum of black hole entropy and ordinary entropy outside the hole does not decrease. Arguing from the GSL, Bekenstein [3] has proposed the existence of a universal bound on the entropy $S$ of any object of maximal radius $R$ and total energy $E$ :

$$
S \leq \frac{2 \pi R E}{\hbar c}
$$

This bound was inferred from the requirement that the GSL be respected when a box containing entropy is deposited with no radial motion next to the horizon of a Schwarzschild black hole (for a Kerr black hole the condition is more complex), and then allowed to fall in. The box's entropy disappears but an increase in black hole entropy occurs. The second law is respected provided $S$ is bounded as in Eq. (1). Other derivations of the bound based on black holes have been given by Zaslavskii [4. [5] and by Li and Liu [6]. Bound (11) can also be interpreted as a bound on the information capacity of any object with total energy $E$ and circumscribing radius $R$ [7, 8 .

Bound (11) has been checked directly for quantum fields enclosed in boxes of various shapes, when the $S$ is interpreted as the logarithm of the number of field quantum states up to energy $E$ above the ground state (for a review see Ref. [9]). Numerical checks have been made for free scalar, electromagnetic and massless spinor fields enclosed in rectangular or spherical boxes [8]. And an analytic proof of the bound for those same free fields valid for boxes of arbitrary shape and topology has been provided [10]. A couple of checks exist for self-interacting fields [9, 11]. All the above demonstrations can be supplemented by the observation that if the box the fields are enclosed in is reckoned as part of the system, the bound is even more strongly satisfied because $E$ is thus augmented while $S$ is hardly changed.

\footnotetext{
*Based on a talk at the Conference on Quantum Aspects of Black Holes, ITP, Santa Barbara, June 1993.

†Electronic mail: bekenste@vms.huji.ac.il

${ }^{\ddagger}$ Permanent address.
} 
Black holes play no part in any of the above considerations. Therefore, bound (1) is known to be true independently of black hole physics for a variety of systems in which gravity is negligible.

By contrast, just a few pieces of evidence exist concerning bound (11)'s validity for self-gravitating systems [4, 12, 13. To these we may add that black holes themselves comply with bound (1) if $R$ in the formula is interpreted as $(\mathcal{A} / 4 \pi)^{1 / 2}$, where $\mathcal{A}$ is the horizon area [3]. Thus our confidence in bound (11) for gravitating systems rests almost entirely on the black hole arguments of Refs. [3 6 .

In Ref. [3] it was assumed that the energy at infinity added to the black hole with the box (which determines the increase in black hole entropy) is that which may be inferred from the redshift factor at the deposition point. However, Unruh and Wald (UW) [14 15 have pointed out that when the deposition is attempted by lowering the box from far away, buoyancy in the radiative black hole environs will prevent lowering the box down to the horizon (if one does not wish to invest energy by pushing it in), and the box will "float" at some neutral point. The total energy at infinity added to the hole after the box has been dropped from the neutral point is larger than the redshifted proper energy of the box. Accordingly, UW concluded that the GSL is respected when the box is dropped, provided only its entropy is restricted by

$$
S \leq V s(E / V),
$$

where $s(e)$ is the entropy density as function of energy density $e$ of unconfined thermal radiation. UW thus concluded that bound (11) is unecessary for the proper functioning of the GSL. They further argued that that bound (11) could not possibly remain correct when the number of particle species in nature is arbitrarily large.

Although it seems reasonable that thermal radiation maximizes entropy as a function of energy density, the UW entropy restriction (2) can easily fail for a system in which surface effects are sizeable, because such a system cannot be described entirely in terms of extensive or intensive variables like $V$ or $E / V$, respectively. In effect, the shape of the system is also a variable. For example, in a rectangular box with dimensions $d \times d \times 0.1 d$, radiation in the energy range $[\hbar / d, 80 \hbar / d]$ explicitly exceeds the UW restriction on entropy [16]. [A more dramatic violation occurs for a black hole; Eq. (2) predicts a bound on $S$ that rises like $E^{3 / 2}$ whereas black hole entropy grows like $E^{2}$. In light of this, UW [15] conjectured that the entropy restriction (2) applies only for box and contents together. In this revised form the restriction may be generally correct for non-gravitating systems; however, it did not play a role in UW's subsequent discussion of the functioning of the GSL in an alternative gedanken experiment (box emptied into black hole and withdrawn open) [15], and its status has remained unclear. And because UW's rescue of the GSL by an appeal to buoyancy [14] in the original gedanken experiment [3] relied on the UW entropy restriction, that experiment has continued to be problematic for the GSL.

In light of the above, we retrace in Sec. II.A UW's analysis of the original gedanken experiment in which a box full of entropy is lowered towards and then dropped into a black hole from the neutral point. For later convenience we perform the gedanken experiment with a Reissner-Nordström $(\mathrm{RN})$ black hole. We studiously avoid UW's approximation that the ambient buoyant pressure varies slowly across the box because it has become clear [17,5, 6] that the neutral point lies very near the horizon where this approximation must fail because of the large gradients. Our exact treatment shows that the UW entropy restriction (2) is neither sufficient nor necessary for the satisfaction of the GSL.

This situation motivates our rederivation of entropy bound (1) which takes full account of UW buoyancy. Our new analysis closely parallels UW's original one, much more so than alternative ones proposed in the wake of UW's paper [17, 16, [5.6]; we pinpoint the stage at which the analysis departs from UW's. In Sec. II.B we determine the position of the neutral point, confirming that it lies very near the horizon. As a result, the buoyancy makes only a small change in the energy bookeeping. The original entropy bound, Eq. (11), is recovered in Sec. II.C.

As mentioned, the independence of bound (1) on the number of particle species has been regarded as a sign that it must fail when the number of species is large [14]. In Sec. III.A we show by explicit calculation of thermal radiation entropy in the thermodynamic limit that the bound is respected for a box containing an arbitrarily large number of massless particle species, provided care is taken to include the vacuum energy in the energy of the full system. We also show, in Sec. III.B, that cavity thermal radiation in the thermodynamic regime in $D$ dimensional space also respects the bound regardless of the value of $D$.

Finally, in Sec. IV we show that the bound strongly restricts the information capacity of the posited black hole remnants, so that they cannot serve to resolve the information paradox.

Henceforth we use units with $G=c=k_{\text {Boltzmann }}=1$, but continue to display $\hbar$.

\section{DERIVATION OF THE ENTROPY BOUND}




\section{A. Critique of Unruh and Wald's Analysis}

The system of interest is a macroscopic rectangular box of total energy $E$ which holds entropy $S$. We label the horizontal crossectional area of the box $A$ and its height $b$. The box is to be lowered towards a black hole with a face facing it in a standard orientation which is defined by two technically convenient conditions. First, we require that $b$ not be very small compared to $A^{1 / 2}$. If this turns out to be the case for the initial orientation, the box is to be rotated by $90^{\circ}$ about a horizontal axis so that a longer edge is brought to vertical orientation, and that edge is to be labeled by $b$. Further, we require that the center of mass (CM) of the box lie initially on the centroid plane (horizontal plane halfway up the box), or below it. If this condition is not satisfied, the box is to be turned upside down (which respects the previous arrangement), and lowered in that orientation. We denote by $R$ the circumscribing radius of the box.

For convenience we carry out the gedanken experiment with a RN black hole of mass $M$ and (not necessarily electric) charge $Q$. The exterior metric may be written as

$$
d s^{2}=-\chi^{2} d t^{2}+\chi^{-2} d r^{2}+r^{2}\left(d \theta^{2}+\sin \theta^{2} d \phi^{2}\right)
$$

where $r$ denotes the usual Schwarzschild radial coordinate and

$$
\chi^{2}=\frac{\left(r-r_{+}\right)\left(r-r_{-}\right)}{r^{2}},
$$

with $r_{ \pm} \equiv M \pm \sqrt{M^{2}-Q^{2}}$. The event horizon lies at $r=r_{+}$and has area $\mathcal{A}=4 \pi r_{+}^{2}$ and entropy $S_{\mathrm{bh}}=\mathcal{A} /(4 \hbar)$. In what follows we also employ the radial proper length measured from the event horizon, $l$, defined by $d l=\chi^{-1} d r$, and use the notation $\Delta=r_{+}-r_{-}$. In order that the box may ultimately be dropped into the hole we assume that

$$
A \ll r_{+}^{2} ; \quad b \ll r_{+} .
$$

We shall assume that the box and its contents are "transparent" to the gauge field of the hole; thus we do not worry about buoyancy due to stresses of this field.

Following UW we assume the black hole has reached equilibrium with its own Hawking radiation, the whole system being enclosed in a large cavity. The black hole temperature $T_{\mathrm{bh}}$ and the local temperature $T$ are related by

$$
T=\frac{T_{\mathrm{bh}}}{\chi}=\frac{\hbar \Delta}{4 \pi r_{+}^{2} \chi} .
$$

The redshift factor $\chi$ enters here as in any equilibrium situation in a gravitational field.

Applying the first law of thermodynamics to a parcel of equilibrium radiation, and assuming that its proper enrgy density $e$, pressure $P$, and proper entropy density $s$ are all functions only of $T$, UW derived the relation

$$
e+P-T s=0
$$

which merely says that the Gibbs free energy of the parcel vanishes, as befits a collection of photons or a mixture of equal numbers of neutrinos and antineutrinos. Differentiation of Eq. (7), use of $T=d e / d s$, and simplification with help of Eqs. (6) and (7) gives

$$
d(P \chi)=-e d \chi
$$

which is equivalent to the condition of hydrostatic equilibrium [14].

The buoyant force acting on the box, as measured by an observer at infinity, is the difference of the redshifted local forces acting on the upper and lower faces [14]:

$$
f_{\text {buoy }}(l)=A\left[(P \chi)_{l-b / 2}-(P \chi)_{l+b / 2}\right]
$$

where $l$ is the proper height of the centroid plane of the box above the horizon. UW approximated the difference in this equation by the first term in a Taylor series. An exact expression follows from using Eq. (8) to convert Eq. (9) to

$$
f_{\text {buoy }}(l)=A \int_{l-b / 2}^{l+b / 2} e \frac{d \chi}{d l^{\prime}} d l^{\prime} .
$$

Let us now express the gravitational force acting on the box and contents. The 4-acceleration of a point with $4-$ velocity $u^{\alpha}$ is defined as $a^{\alpha}=u_{; \beta}^{\alpha} u^{\beta}$. A simple calculation shows that a point stationary in Schwarzschild coordinates has invariant acceleration 


$$
a \equiv\left(a^{\alpha} a_{\alpha}\right)^{1 / 2}=\chi^{\prime}
$$

where a prime denotes a derivative with respect to $r$. We can thus write the "gravitational force" acting on the box as measured at infinity in the form

$$
f_{\text {grav }}(l)=-A \int_{l-b / 2}^{l+b / 2} \rho \frac{d \chi}{d l^{\prime}} d l^{\prime}
$$

Here $\rho$ denotes the proper energy density of the box and contents, and $d \chi / d l=a \chi$ is the local acceleration as measured at infinity. The minus sign reminds us that gravity and buoyancy act in opposite senses.

Putting together Eqs. (10) and (12) we write the work done by the box on the agent lowering it from infinity down to proper height $l$ above the horizon as

$$
W(l)=\int_{\infty}^{l}\left(f_{\text {buoy }}+f_{\text {grav }}\right) d l^{\prime} .
$$

$W(l)$ is maximized when the box's centroid plane reaches the neutral point, $l=l_{0}$. Setting $d W / d l=0$ we obtain the condition determining $l_{0}$ :

$$
f_{\text {buoy }}\left(l_{0}\right)+f_{\text {grav }}\left(l_{0}\right)=A \int_{l_{0}-b / 2}^{l_{0}+b / 2}(e-\rho) \frac{d \chi}{d l} d l=0 .
$$

Henceforth, we shall adopt the following notation for integrals like those appearing in Eqs. (10), (12) and (14):

$$
A \int_{l_{0}-b / 2}^{l_{0}+b / 2} F d l \Longleftrightarrow \int_{V} F d V
$$

where $d V$ stands for the element of box volume $A d l$. Note that if $e$ and $d \chi / d l$ are nearly constant across the box, the condition (14) may be approximated by UW's form, c.f., Eq. (2.2) of Ref. [14],

$$
e V=E \equiv \int_{V} \rho d V
$$

where $V=A b$ is the box's volume.

Since $W(l)$ is maximum at $l=l_{0}$, the mass increment $\delta M$ of the black hole is minimal if the box is dropped in from the neutral point. Evidently $(\delta M)_{\min }=E-W\left(l_{0}\right)$. Using Eq. (13) we may reeexpress this as

$$
(\delta M)_{\min }=\int_{V} \rho \chi d V+\int_{V} P \chi d V .
$$

The first integral is just the energy at infinity of the box, with each parcel properly redshifted. This integral equals $E-\int_{\infty}^{l_{0}} f_{\text {grav }} d l$. The second integral is just $-\int_{\infty}^{l_{0}} f_{\text {buoy }} d l$ with $f_{\text {buoy }}(l)$ in the form (9). As a result of the cancellation of the work done by buoyant forces on top and bottom of the box over the range $\left[l_{0}+b / 2, \infty\right]$, the buoyant contribution to $(\delta M)_{\text {min }}$ only depends on the distribution of $P \chi$ over the height of the box at the neutral point.

UW's version of Eq. (17), namely

$$
(\delta M)_{\min }=(E+P V) \chi
$$

would follow from Eqs. (16) and (17) if $P$ and $\chi$ separately varied little across the box. Because the neutral point turns out to be so near the horizon (see Sec. II.B), these quantities actually vary a lot across the box in the generic case, so that the approximations leading to Eq. (18) are questionable.

Instead, let us replace $P$ in Eq. (17) by means of the identity (7). Taking cognizance of Eq. (6) we get

$$
(\delta M)_{\min }=\int_{V}(\rho-e) \chi d V+T_{\mathrm{bh}} \int_{V} s d V .
$$

Were $\chi$ to be nearly constant across the box, the first integral here would vanish by virtue of Eq. (16), and we would be left with UW's expression (2.22) of Ref. 14. Since taking $\chi$ as nearly constant is an unwarranted approximation in view of the closeness of the neutral point to the horizon, let us instead use Eqs. (19) and (6) to compute the overall entropy change of the world, $(\delta S)_{\text {tot }} \equiv(\delta M)_{\min } / T_{\mathrm{bh}}-S$, when the box is dropped from the neutral point. We find 


$$
(\delta S)_{\mathrm{tot}}=T_{\mathrm{bh}}^{-1} \int_{V}(\rho-e) \chi d V+\int_{V} s d V-S .
$$

In UW's discussion, the first integral in Eq. (20) dropped out in wake of the indicated approximations. It may be seen by comparing with Eq. (14) that, in fact, its magnitude and even its sign depend on the distribution of $e$ and $\rho$ across the box. Thus, in contrast with UW's discussion, we must conclude that validity of the UW entropy restriction, Eq. (2), does not by itself guarantee that the GSL will be obeyed. And conversely, assuming that the GSL is satisfied in the gedanken experiment in question, does not allow us to derive the UW entropy restriction for the box. Unless supplemented by detailed information about the box, the entropy restriction is neither a necessary nor a sufficient condition for the operation of the GSL. Therefore, it seems best to calculate in detail the sum of integrals in Eq. (20).

\section{B. The Neutral Point}

As the first step we make an approximate determination of the location of the neutral point by using UW's criterion Eq. (16), and a model of radiation as a mixture of noninteracting gases of massless particles, one for each species in nature. According to Boltzmann, for such radiation

$$
P=e / 3=\frac{N \pi^{2} T^{4}}{45 \hbar^{3}}
$$

where $N$ is the effective number of particle species (photon and graviton contribute one to $N$, while each neutrino and antineutrino species counts as $7 / 16)$.

With Eq. (6) for $T$, criterion (16) amounts to

$$
\chi\left(l_{0}\right)=0.0717 N^{1 / 4}(A b \hbar / E)^{1 / 4} \Delta r_{+}^{-2} .
$$

Since we assume the box to be macroscopic, and hence large compared to its Compton wavelength, $\hbar / E \ll b$. This together with inequalities (5) tells us that for realistic values of $N$ (in Sec. IIIA we shall reconsider the situation where $N$ is arbitrarily large), $\chi\left(l_{0}\right) \ll \Delta r_{+}^{-1}$. A look at Eq. (四) shows that the neutral point must lie in the region $r-r_{+} \ll \Delta$. But in this region, a good approximation to the proper distance from the horizon to a point $r$ is

$$
l \approx 2 r_{+}\left(r-r_{+}\right)^{1 / 2} \Delta^{-1 / 2} .
$$

It is clear that in that region $l \ll r_{+}$. In the same approximation Eq. (4) can be written

$$
\chi(l) \approx \frac{1}{2} \Delta r_{+}^{-2} l .
$$

Two important consequences follow from the linear form of $\chi$ in the near horizon region where the neutral point lies. First from Eq. (6)

$$
T=\hbar /(2 \pi l),
$$

so that, as a function of proper distance from the horizon, $T$ does not depend on the black hole's parameters. This universal form for $T$ may be understood by writing down the Unruh temperature for an (accelerated) sationary observer, $T_{\mathrm{U}}=\hbar a /(2 \pi)$, and using Eqs. (11) and (24) to recast it into a form identical to Eq. (25). The equality $T=T_{\mathrm{U}}$ in the near horizon region allows one to interpret the thermal radiation in equilibrium with the hole as observer dependent Unruh acceleration radiation seen by the stationary observers. One may thus follow UW in asserting that the large $e$ and $P$ corresponding to $T$ at the neutral point do not generate strong curvature, i.e., that the metric (3) remains an excellent approximation.

The second consequence of the $\chi \propto l$ form is that condition (14) can be rewritten as

$$
\int_{V} e d V=E
$$

which, unlike the UW condition (16), is accurate in the near horizon region, and indeed becomes the more accurate the nearer to the horizon since Eq. (24) is asymptotically exact there.

Let us now use Eq. (26) to accurately determine $l_{0}$. Substituting $e$ from Eq. (21) and $T$ from Eq. (25), and remembering that the range of $l$ in the integral is $\left[l_{0}-b / 2, l_{0}+b / 2\right]$, the condition leads to 


$$
\frac{\left(l_{0}^{2}-b^{2} / 4\right)^{3}}{3 l_{0}^{2} b^{4}+b^{6} / 4}=\eta^{3} \equiv \frac{N \hbar A}{720 \pi^{2} E b^{3}}
$$

In our world where the number of particle species that would be excited by a massive black hole is limited, $\eta \ll 1$ because $\hbar / E \ll b$ and because in the standard box orientation, $b^{2}$ cannot be very small compared to $A$. In Sec. III.A we shall argue that $\eta \ll 1$ remains true even when $N$ is arbitrarily large.

When $\eta \ll 1$, it follows from Eq. (27) to lowest order in $\eta$ that

$$
l_{0}^{2} \approx(1 / 4+\eta) b^{2} .
$$

Therefore, at the neutral point, the centroid plane of the box is just a little farther from the horizon than half the box's height, which means the box floats almost touching the horizon. This phenomenon has been noted earlier [17,5,6]. Since the neutral point does occur near the horizon, the approximations involved in Eqs. (23) and (24) are seen to be justified a posteriori. The finding here serves to emphasize that the UW neutral point condition (16) cannot be accurate because of the strong gradient of $e$ near the horizon, and should properly be replaced by condition (26).

Of course, all the above hinges on the correctness of the Boltzmann model, Eq. (21). Although the appearance of new species as $T$ rises is known not to cause a measurable departure from the Boltzmann "equation of state", but merely requires that $N$ be regarded as slowly rising, at sufficiently high $T$ interactions may cause a substantial departure from the simple relation (21). How would this affect our discussion?

Let us, in the manner of high energy physics, characterize the onset of a strong departure from the Boltzmann model by a scale of length $L$ which is connected to the appropriate transition temperature by a relation much as Eq. (25). We know that $L<10^{-12} \mathrm{~cm}$ because Eq. (21) works well in cosmology all the way back to the lepton era. If the strong interaction is the one that spoils the Boltzmann model, we may expect $L \leq 10^{-13} \mathrm{~cm}$. At any rate, it is clear that $L$ is much smaller than the dimensions of a macroscopic box.

A departure from Boltzmann's model affects our discussion only if at the neutral point the lower side of the box has penetrated to within $l<L$, so that it is exposed to temperatures beyond the transition. But, of course, since the box is much larger than $L$, this means that at the neutral point the box must nearly touch the horizon, with its centroid plane at proper height $\approx b / 2$, just as we found by relying on the Boltzmann model. Thus, as far as the location of the neutral point is concerned, complications beyond the Boltzmann model are of no practical consequence.

\section{The Entropy Bound}

Proceeding with our program, we now compute explicitly $\delta S_{\text {bh }}$ by going back to Eq. (17). Due to the linearity of $\chi$ with $l$, the integral over $\rho$, the energy density in the box, may be expressed in terms of the proper height of its CM above the horizon, $l_{\mathrm{cm}}$, defined by $\frac{1}{2} \Delta r_{+}^{-2} l_{\mathrm{cm}}=\bar{\chi} l_{l_{0}}$ (average with respect to $\rho$ ), and $E$ as defined by Eq. (16). And the integral over $P$ may be worked out with help of Boltzmann's model, Eq. (21), and Eqs. (23)- (25). The result is

$$
(\delta M)_{\min }=\frac{1}{2} \Delta r_{+}^{-2} E\left[l_{\mathrm{cm}}+\eta^{3} l_{0} b^{4}\left(l_{0}^{2}-b^{2} / 4\right)^{-2}\right] .
$$

A look at Eq. (28) shows that the square parenthesis in Eq. (29) amounts to $l_{\mathrm{cm}}+\eta l_{0}$; the second term comes from the buoyancy. We recall now that in the standard box orientation employed here, the CM of the box cannot lie above the centroid plane $\left(l_{\mathrm{cm}} \leq l_{0}\right.$ initially, and the CM can only slip down as the box enters strong gravitational fields). And because $l_{0} \approx b / 2+\eta b$ [see Eq. (28)], the square parenthesis in Eq. (29) cannot exceed $(1+3 \eta) b / 2$. The buoyancy corrections of $\mathrm{O}(\eta)$ are evidently negligible under the same assumptions that led us to conclude that the neutral point is near the horizon. We thus obtain

$$
(\delta M)_{\min } \leq \frac{1}{4} \Delta r_{+}^{-2} b E
$$

which is another version of Eq. (19).

From Eq. (30) we may compute, with help of the first law for black holes, Eq. (6) and the obvious constraint $b<2 R$ that when the box is dropped from the neutral point,

$$
(\delta S)_{\text {tot }}<2 \pi R E / \hbar-S,
$$

which replaces Eq. 20). It is evident from this inequality that in order for the GSL to be satisfied $\left[(\delta S)_{\text {tot }} \geq 0\right]$, the box entropy $S$ must satisfy the bound on entropy (1). 
The above argument requires modification if at the neutral point the box already penetrates into the region with $T$ beyond the transition temperature at which the Boltzmann model gets modified. We first note that whatever the true relation between $e, P$ and $T$, the inequality $P<e$ must be satisfied. The reason is that causality demands that $d P / d e \leq 1$ (speed of sound in the radiation subluminal) for all $T$. Integrating the inequality $d P-d e \leq 0$ from low $T$ where $P=e / 3$ certainly applies, we see that $P<e$ for any $\mathrm{T}$, however large. Consider now the identity

$$
d(P \chi l)=l d(P \chi)+P \chi d l
$$

If we substitute in it from Eq. (8), and take into account that in the near horizon region $l d \chi=\chi d l$, we see that $P<e$ implies that $d(P \chi l) / d l<0$ near the horizon. This means that $P(l)$ drops off with $l$ faster than $l^{-2}$, which means that whatever the modification to the Boltzmann model, $P(T)$ must grow faster than $T^{2}$.

In light of the above, consider the buoyant contribution to $(\delta M)_{\min }$ in Eq. (17), written as

$$
\int_{V} P \chi d V=\frac{\int_{V} P \chi d V}{\int_{V} P d V} \cdot \int_{V} P d V .
$$

By the causality constraint, $\int_{V} P d V<E$ [see Eq. (26)]. The ratio of integrals is evidently largest when $P$ decreases slowest with $l$. We may thus bound from above that ratio by using the limiting form $P \propto l^{-2}$ to compute it. Recalling Eq. (15) we thus have

$$
\int_{V} P \chi d V<\frac{1}{2} \Delta r_{+}^{-2} E\left(l_{0}+b / 2\right) \frac{\ln y}{y-1},
$$

where $y \equiv\left(l_{0}+b / 2\right)\left(l_{0}-b / 2\right)^{-1}$. The closeness of the box's bottom to the horizon at the floating point means that $l_{0} \approx b / 2 ;$ therefore, $l_{0}+b / 2 \approx 2 l_{0}$ and $y \gg 1$. Hence,

$$
\int_{V} P \chi d V=\varpi \Delta r_{+}^{-2} l_{0} E,
$$

where $\varpi<\ln y /(y-1) \ll 1$.

Inserting this result in Eq. (17) for $(\delta M)_{\min }$ and recalling that the integral over $\rho$ is already evaluated in our previous result 29 , we have

$$
(\delta M)_{\min }=\frac{1}{2} \Delta r_{+}^{-2} E\left[l_{\mathrm{cm}}+2 \varpi l_{0}\right]
$$

Again, because the box is standardly oriented and very close to the horizon, $l_{\text {cm }} \leq l_{0} \approx b / 2 \leq R$. And because $\varpi \ll 1$, the buoyant term evidently cannot make the square brackets larger than $R$. We thus find that Eqs. (30) and (31) apply again, and the entropy bound (1) follows from the assumed validity of the GSL, as in the case when the Boltzmann model could be used down to the neutral point.

In all arguments in this subsection, a slightly tighter bound on entropy would follow if we worked throughout in terms of $b$, rather than appealing to the inequality $b<2 R$. The impression we get from this that the maximal entropy of a thin box decreases with decreasing thickness $b$ is supported by numerical computations [8] if $b$ is not too small. Those calculations do not support Li and Liu's conclusion [6] that the entropy bound is unconditionally set by the smallest box dimension. After all, in 3-dimensional space a 2-dimensional box filled with massless quanta can hold nonvanishing entropy. In our approach here the bound could not be derived in terms of $b$ for arbitrarily small $b$ because the condition $\eta \ll 1$ will fail when $b$ gets sufficiently small [see Eq. (27)].

\section{EXTENSIONS}

\section{A. Irrelevance of Species Number}

Our derivation of the bound in Sec. II assumed $\eta \ll 1$, which condition would seem to fail in a world where $N$ is very large. Indeed, UW contended that no bound of type (1), which is independent of the number of species, could possibly retain its validity as $N$ becomes large because it is known that the more species there are, the larger the number of states (entropy) accessible with given energy. Their own entropy restriction, Eq. (2), scales as $N^{1 / 4}$, that being the dependence of Boltzmann's formula for blackbody radiation entropy at given energy on the number of species. UW conceived of buoyancy, with its intrinsic dependence on the number of species in the radiation, as 
nature's exclusive way to defend the GSL against a violation when a body made up of many species (large entropy with moderate energy) is lost down a black hole.

That this view must have restricted validity is clear from the following argument demonstrating that an $N-$ independent entropy bound must exist in order for the GSL to function, even in situations where buoyancy cannot play a role.

Consider a box with energy $E$ and entropy $S$ dropped freely from far away into an exactly extremal RN black hole of mass $M$ in empty space. Because the fall is free i.e., geodesic, the box does not feel Unruh radiation. And because the hole is extremal, thermal radiant pressure is absent. Hence there is no buoyancy to complicate the energy bookeeping. Initially $S_{\mathrm{bh}}=\pi M^{2} / \hbar$. After the black hole has assimilated the box, the mass has gone up to $M+E$ and $S_{\mathrm{bh}}=\pi\left(M+E+\sqrt{2 M E+E^{2}}\right)^{2} / \hbar$ (because $Q$ is unchanged). We regard $E$ as a small quantity. Then to lowest order in $E$,

$$
\delta S_{\mathrm{bh}}=\pi(2 M)^{3 / 2} E^{1 / 2} / \hbar .
$$

Coherent gravitational and electromagnetic radiation may be emitted due to the infall. The energy in these radiations is expected to be of order $E^{2} / M$ [20. Its substraction from the final $M$ will produce terms in Eq. (37) of the same order as those we have already discarded. Thus the infall radiations prove negligible, and in any case they would act to reduce $\delta S_{\mathrm{bh}}$. There may also be some radiation entropy emitted by the Hawking process as the box is assimilated and the black hole departs from exact extremality. Once the black hole is not extreme, it should radiate entropy at a rate a bit higher than a blackbody of temperature $T_{\mathrm{bh}}$ and area $\mathcal{A}$ [19. This entropy emission should be somewhat larger than the induced decrease in $S_{\mathrm{bh}}$. Therefore, the overall entropy growth rate would be

$$
d S_{\mathrm{bh}} / d t+d S_{\mathrm{rad}} / d t \sim(180)^{-1} \Delta^{3} r_{+}^{-4} .
$$

In our case, after the assimilation $\Delta=2(2 M E)^{1 / 2}$. Therefore, over a period of coordinate time $\sim M$, which is of the order of the time required for the effective disappearance of the box, the just perturbed black hole should generate entropy $\sim(4 \sqrt{ } 2 / 45)(E / M)^{3 / 2}$, which is certainly much smaller than $\delta S_{\mathrm{bh}}$ because $M$ has to be large compared to the Planck mass.

Thus we have accounted for all entropy contributions. In order for the GSL not to be violated, the box's entropy $S$ must be bounded by $\pi(2 M)^{3 / 2} E^{1 / 2} / \hbar$. Of course, for large $M$ this is a much larger bound than Eq. (1). However, the point is that the new bound, though derived without recourse to lowering into the black hole, is also independent of $N$. This shows that there must exist an $N$-independent bound on the entropy of a bounded system, arguments involving buoyancy notwithstanding.

This said, we still face a paradox: by Boltzmann's formulae it seems that for large enough $N$, a quantity of radiation with given energy should surpass any $N$-independent entropy bound. The resolution we develop here depends on recognizing that the bounds on entropy derivable with the help of black holes must always refer to an entire system, not to part of one. In particular, bound (11) refers to the entropy and energy of the box and its contents. Therefore, when comparing thermal radiation entropy in a box with the bound, one would like to restate the Boltzmann formula for entropy in a form that takes cognizance of the existence of the box and those of its properties which are responsible for confining the radiation. Then a comparison can be made.

We shall perform the concrete calculations for a spherical box of radius $R$ and total energy $E$. According to Boltzmann, at temperature $T$ thermal radiation in the box has energy

$$
E_{\mathrm{rad}}=\left(4 \pi^{3} / 45\right) N R^{3} T^{4} \hbar^{-3}
$$

and entropy

$$
S_{\mathrm{rad}}=\left(16 \pi^{3} / 135\right) N R^{3} T^{3} \hbar^{-3} .
$$

If $E_{0}$ stands for the energy of the empty box $(E$ at $T=0)$, then upon eliminating $T$ we have

$$
S=(4 \sqrt{ } 2 / 135) N^{1 / 4}\left[45 \pi R\left(E-E_{0}\right) / \hbar\right]^{3 / 4} .
$$

How small can $E_{0}$ be ? Even when empty, the box's energy receives a contribution from vacuum (Casimir) energy of those fields it can entrap (or keep out). On dimensional grounds each species contributes vacuum energy $\varepsilon=\alpha \hbar / R$ where $\alpha$ is dimensionless. For the electromagnetic field in a sphere Boyer [21] showed that $\alpha=0.045$. For all species together we write $\varepsilon=\bar{\alpha} N \hbar / R$, where $\bar{\alpha}$ is the suitable average. If positive, the vacuum energy sets a lower bound on $E_{0}$.

Even when the vacuum energy is negative [22,23], the total box mass must end up being positive. The physical mechanism is the suction on the box's wall that must accompany negative vacuum energy. In fact, from the rate 
at which $\varepsilon$ gets more negative as the box's radius decreases, it follows that the wall sustains a negative pressure $P=-\bar{\alpha} N \hbar / 4 \pi R^{3}$. To resist this suction the wall must maintain a surface pressure (force per unit length) of the same order [17. But unless the wall's surface mass density is bigger than the surface pressure, the speed of sound in the wall would be superluminal. The conclusion is that the wall must have a mass comparable to the magnitude of $\varepsilon$, or larger. This will make $E_{0}$ positive. Since an exact cancellation between wall mass and vacuum energy is unlikely, we expect $E_{0}$ to be of order $|\bar{\alpha}| \hbar / R$, or larger. Thus, henceforth, we simply write $E_{0}=\alpha N \hbar / R$ and assume $\alpha>0$.

The formula for entropy, Eq. (41) is now

$$
S_{\mathrm{rad}}=(4 \sqrt{ } 2 / 135) N^{1 / 4}[45 \pi(R E / \hbar-\alpha N)]^{3 / 4} .
$$

Now the function $f(x)=(x-a)^{3 / 4} / x$ has a maximum value of $\frac{1}{4} 3^{3 / 4} a^{-1 / 4}$. We may, therefore, deduce from Eq. 42 ) the inequality

$$
S_{\mathrm{rad}} \leq \frac{\sqrt{ } 2 \pi^{3 / 4}}{(135 \alpha)^{1 / 4}} \frac{R E}{\hbar}
$$

This bound for blackbody radiation in a box is of the same form as bound (11). Indeed, since typically $\alpha \sim 10^{-3}-10^{-2}$ for a single field [22,23], the numerical coefficient here is $\sim 3-5.5$ in harmony with bound (1). Most important, the bound here obtained from statistical physics is independent of $N$, just as (1) is. The physical mechanism is that some of the energy $E$ is inert energy whose magnitude depends on $N: E>E_{0}=\alpha N \hbar / R$. Thus the factor $R E / \hbar$ actually grows with $N$. But the point is that when we state an entropy bound in terms of $R$ and $E$, we do not have to worry about how big $N$ is; the bound will take care of that automatically.

At this junction we return to the issue of the allowed range of $\eta$. We have already seen that when $N$ is a few, and the box is standardly oriented, $\eta \ll 1$. Let us now analyze the case when $N$ is large for a spherical box of radius $R$. Then we may use our result that $E>E_{0}=\alpha N \hbar / R$ in the definition (27) by taking $A=4 \pi R^{2}$ and $b=2 R$. We get

$$
\eta^{3}=\frac{N \hbar}{1440 \pi E R}<\frac{1}{1440 \pi \alpha}
$$

which shows that $\eta$ does not grow indefinitely with $N$. Furthermore, unless $\alpha$ is much smaller than the typical value for separate fields, $\sim 10^{-2}-10^{-3}, \eta$ is indeed small compared to unity, thus allowing the arguments following Eq. (27) to yield the entropy bound from an appeal to black hole physics. And this bound is, of course, consistent with Eq. (43).

The case of a nonspherical box is much harder to analyze because $E_{0}$ depends not only on the typical dimension of the box, but also on axis ratios [22]. However, it seems plausible that $\eta$ will also be small in those cases provided the various box dimensions are not too different.

\section{B. Irrelevance of Many Dimensions}

Not only does the proliferation of particle species increase the entropy for given energy, proliferation of spatial dimensions has the same effect. Evidently the more the dimensions, the more ways there are to split up the energy, so that a higher entropy is obtained. We might thus naively expect a bound like (11) to be violated as the number of dimension increases without bound. As we now show, this conclusion would be premature. Consider in $D$ flat spatial dimensions a spherical space of radius $R$ into which we dump energy $E$. What can we say about the entropy $S(E)$ as $D$ grows ? Evidently maximal $S(E)$ corresponds to the excitation of all existing field species with like inverse temperature $\beta$. The description of our fixed energy system in terms of temperature (thermodynamic regime) is tenable provided $E$ is large enough that energy fluctuations in the canonical ensemble for $\beta$ are small. In practice this means $\beta \hbar / R \ll 1$ (many wavelenghts small compared to $R$ are thermally excited), which we assume to be true. We shall simplify matters by ignoring massive species. This corresponds to the case that $\beta$ times any of the rest masses is large.

The volume of a sphere of radius $r$ in $D$ dimensions is [24]

$$
V_{D}(r)=\frac{2 \pi^{D / 2} r^{D}}{D \Gamma(D / 2)},
$$

where $\Gamma$ denotes the Euler gamma function. Consequently, the volume in frequency space of the shell $(\omega, \omega+d \omega)$ is

$$
d V_{D}(\omega)=D\left[V_{D}(\omega) / \omega\right] d \omega
$$


The mean thermal energy in the sphere from one helicity degree of freedom is

$$
E=V_{D}(R) \int_{0}^{\infty} \frac{\hbar \omega d V_{D}(\omega)}{\left(e^{\beta \hbar \omega} \mp 1\right)(2 \pi)^{D}},
$$

where upper (lower) signs correspond to boson (fermion) fields. Using Eqs. (45,46), and

$$
\int_{0}^{\infty} \frac{x^{D} d x}{e^{x} \mp 1}=\zeta(D+1) \Gamma(D+1) \times \begin{cases}1 & \text { for bosons; } \\ 1-2^{-D} & \text { for fermions } .\end{cases}
$$

where $\zeta(z)$ is Riemann's zeta function, we can cast the mean energy of all massless species in the form

$$
E=\frac{N \zeta(D+1) \Gamma(D) R^{D}}{2^{D-3}[\Gamma(D / 2)]^{2} \beta^{D+1} \hbar^{D}},
$$

where $N$ is the number of massless species (massless nonscalar bosons contribute one to $N$, while massless fermions contribute $\left.2^{-1}-2^{-(D+1)}\right)$.

Likewise, we can write the thermal entropy of one helicity degree of freedom as

$$
S=V_{D}(R) \int_{0}^{\infty}\left[\mp \ln \left(1 \mp e^{-\beta \hbar \omega}\right)+\frac{\beta \hbar \omega}{e^{\beta \hbar \omega} \mp 1}\right] \frac{d V_{D}(\omega)}{(2 \pi)^{D}} .
$$

After integration by parts, the first term in square brackets is seen to reduce, by virtue of Eq. (46), to $D^{-1}$ times the second. Comparing the result for the entropy of all helicity degrees of freedom with Eq. (47) we see that

$$
S_{N}=(1+1 / D) \beta E .
$$

The special case for $D=3$ of this equation is well known.

As mentioned, the condition $\beta \hbar / R \ll 1$ must be respected in order for the above continuum treatment to make sense. Solving Eq. (49) for the relevant quantity we have

$$
\beta \hbar / R=C_{D}(N \hbar / R E)^{\frac{1}{D+1}},
$$

with

$$
C_{D} \equiv\left[\frac{\zeta(D+1) \Gamma(D)}{2^{D-3}[\Gamma(D / 2)]^{2}}\right]^{\frac{1}{D+1}} .
$$

Numerically it is found that $C_{D}$ decreases with $D: C_{D}=(1.34,1.29,1.25, \ldots, 1)$ for $D=(1,2,3, \ldots, \infty)$. Hence the condition on $\beta$ amounts to $R E / N \hbar \gg 1$. So, just as in Sec. III.A, we find here that $N$ constrains the value of $R E$. Substituting Eqs. (52 53) into Eq. (51) we get

$$
S_{N}=C_{D}(1+1 / D) N^{\frac{1}{D+1}}(R E / \hbar)^{\frac{D}{D+1}} .
$$

The case $D=3$ of this should be compared with Eq. (42); the only real difference is that here $E$ does not include vacuum energy.

Let us now take the limit $D \rightarrow \infty$ while keeping $N$ fixed. In this limit $\zeta(D+1) \rightarrow 1$ and $\Gamma(D+1) \rightarrow \sqrt{2 \pi} e^{-D} D^{D}$, so we find rigorously that

$$
\lim _{D \rightarrow \infty} S_{N}=R E / \hbar
$$

Thus despite the many dimensions, and the arbitrary number of species $N$, this entropy is consistent with bound (1). As in Sec. III.A, the dependence on $N$ has dropped out except for the fact that our end result is valid only if $E$ is large enough on a scale set by $N$. And contrary to intuition, in the thermodynamic limit the large phase space that opens up because of the multiplicity of dimensions does not help the entropy to surpass the entropy bound (1).

What happens for $D$ finite? We rewrite Eq. (54) as

$$
S_{N}=C_{D}(1+1 / D)(N \hbar / R E)^{\frac{1}{D+1}} R E / \hbar .
$$

Numerically $C_{D}(1+1 / D)$ decreases monotonically from 2.89 at $D=1$ to unity as $D \rightarrow \infty$. Because $N \hbar / R E \ll 1$ in the thermodynamic regime, it is clear that the entropy $S_{N}$ conforms to bound (11) for any $D$. We must stress that this result, like the previous one, is not guaranteed if the condition for the thermodynamic regime fails. In that case one cannot rely on continuum formulae like Eqs.(50 51), and must take recourse to numerical calculations of the energy distribution of quantum states in a cavity. As mentioned, for $D=3$ such calculations have been made [8], and fully confirm bound (1). When generalizing these to arbitrary $D$ and $N$, care should be taken to include vacuum energies. 


\section{INFORMATION CAPACITY OF REMNANTS}

Remnants of black holes have been suggested as a resolution of the black hole information paradox (for reviews see Ref. 25]). In one version of this idea [26], the black hole stops evaporating when its dimension approaches the Planck scale. It is hypothesized that this remnant quiescent object retains the large information that went down the initial black hole upon its formation. With a notable exception [27], the arguments for and against Planck scale remnants have not confronted quantitatively the question of how much information can really be contained in a space which looks so small from the outside, and has a correspondingly tiny externally measured mass energy.

Now, if a system contains information $I$ (natural units), then it must have at its disposal at least $n=e^{I}$ internal states. When looked at in coarse grainning, such a system will be ascribed entropy $S=\max (\ln n) \geq I$. Now, the entropy bound (1) must also bound the entropy of a gravitating system since we never specified in Sec. II details about the box's interior. Applied to a remnant the bound would predict that for radius $\ell_{\text {Planck }}$ and mass $\hbar / \ell_{\text {Planck }}$ its entropy is $2 \pi$ at most. The remnants information content is thus just a few bits. This makes Planck-size remnants irrelevant for the information paradox (the system that collapsed to the black hole which fathered the remnant may have been specified by trillions of bits).

Giddings introduced the idea of large remnants 27] to overcome problems like this. The idea is that each black hole decays to a remnant of a different size, which is deemed capable of retaining the initial information. Now, for a Schwarzschild black hole of initial mass $M_{0}$, the initial black hole entropy $S_{\mathrm{bh}}=4 \pi M_{0}^{2}$ measures the total information that becomes hidden at the moment of collapse. As argued above, Eq. (1) should bound the information that can be held by the remnant whose mass and radius are $E$ and $R$. We assume $R \sim 2 E$ because the remnant must be a strongly gravitating object. Thus for the remnant to succesfully retain the initial information, we need $E \geq M_{0}$ which is impossible since some evaporation must have taken place. The point is that black holes saturate the entropy (information) bound so that the equivalent amount of information cannot be held by a lighter strongly gravitating object.

Clearly the role of remnants, Planck size or larger, might be rescued if they were not to respect the entropy bound (11). We have argued that the bound must apply to any object that can be lowered to the horizon of a black hole. Perhaps a remnant, by virtue of its black hole nature, cannot be supported in the requisite way to be lowered. However that may be, any remnant can still be dropped freely into a bigger extreme RN black hole of mass $M$. Repeating the argument at the beginning of Sec. III.A, and arguing that the information-coding states of the remnant contribute towards coarse grained entropy, we see that the procedure bounds the entropy, and hence the information capacity, of the remnant by $\pi(2 M)^{3 / 2} E^{1 / 2}$ (otherwise the GSL gets violated in the remnant black hole merger).

Now $M$ has to be bigger than $E$ so that the remnant can be absorbed, but it does not have to be arbitrarily large. Thus this independent argument bounds the remnant's information capacity by $\gamma E^{2}$ with $\gamma$ perhaps a few times $2^{3 / 2} \pi$. If the remnant has descended from a neutral black hole of initial mass $M_{0}$, it must retain information $16 \pi M_{0}^{2}$ to do its job as information repository in the resolution of the information paradox. This information will conform to the bound we have just set provided $E>4(\pi / \gamma)^{1 / 2} M_{0}$. We see that the remnant cannot be much lighter than $M_{0}$. However, there seems to be no reason for black hole evaporation to turn off as soon as the black hole has lost a moderate fraction of its mass. This problem dramatizes the difficulty the remnant scenario has in resolving the black hole information paradox.

The usual argument for remnants as information repositories is that they can retain large information despite their small dimension because they contain a very large internal space in the form of a throat or horn. According to this argument, a bound like (11), which is stated in terms of external dimensions alone, cannot apply. But we have just seen that if a remnant had an information capacity well above that indicated by bound (1) in terms of its external dimension, it would cause a violation of the GSL, were it dropped into a large black hole. This point might be countered if the infall of the remnant produces a singularity, thus making the GSL moot. However, in that case the whole role of remnants as stable information repositories is put in doubt. The conclusion must be that black remnants, as discussed heretofore, cannot resolve the information paradox.

\section{ACKNOWLEDGMENTS}

I thank Ted Jacobson, Don Page and Bob Wald for a number of remarks, Leonard Parker for suggestions, Jim Hartle for his interest in the subject, Hartle and Gary Horowitz for hospitality in Santa Barbara, and the Aspen Center for Physics where the draft of this paper was completed. 
[1] J. D. Bekenstein, Lett. Nuov. Cim. 4, 737 (1972) and Phys. Rev D7, 2333 (1973); S. W. Hawking, Phys. Rev. D13, 2460 (1976).

[2] J. D. Bekenstein Phys. Rev. D9, 3292 (1974).

[3] J. D. Bekenstein, Phys. Rev. D23, 287 (1981).

[4] O. B. Zaslavskii, Phys. Letters A160, 339 (1991) and Gen. Rel. Grav. 24, 973 (1992).

[5] O. B. Zaslavskii, "Bekenstein entropy upper bound and laws of thermodynamics", Kharkov University preprint, 1993.

[6] L. X. Li and L. Liu, Phys. Rev. D46, 3296 (1992).

[7] J. D. Bekenstein, Phys. Rev. Letters 46, 623 (1980).

[8] J. D. Bekenstein, Phys. Rev. D30, 1669 (1984).

[9] J. D. Bekenstein and M. Schiffer, Int. J. Mod. Phys. C 1, 355 (1990).

[10] M. Schiffer and J. D. Bekenstein, Phys. Rev. D39, 1109 (1989).

[11] J. D. Bekenstein and E. I. Guendelman, Phys. Rev. D35, 716 (1987).

[12] R. Sorkin, R. M. Wald and Z. Z. Jiu, Gen. Rel. Grav.13, 1127 (1981).

[13] W. H. Zurek and D. N. Page, Phys. Rev. D29, 628, (1984).

[14] W. G. Unruh and R. M. Wald, Phys. Rev. D25, 942 (1982)

[15] W. G. Unruh and R. M. Wald, Phys. Rev. D 27, 2271 (1983).

[16] J. D. Bekenstein, Phys. Rev. D27, 2262 (1983).

[17] J. D. Bekenstein, Phys. Rev. D26, 950 (1982).

[18] W. G. Unruh, Phys. Rev. D14, 870 (1976).

[19] D. N. Page, Phys. Rev. D14, 3260 (1976).

[20] M. Davis, R. Ruffini and J. Tiomno, Phys. Rev. 5, 2932 (1972).

[21] T. H. Boyer, Phys. Rev. 174, 1764 (1968).

[22] W. Lukosz, Z. Phys. 262, 327 (1975).

[23] S. D. Unwin, Phys. Rev. D26, 944 (1982).

[24] K. Huang, Statistical Mechanics (Wiley, New York, 1963).

[25] J. Preskill, "Do black holes destroy information ?", hep-th/9209058; J. Harvey and A. Strominger, "Quantum aspects of black holes", hep-th/9209055; S. Giddings, "Toy models for black hole evaporation", hep-th/9209113.

[26] L. Susskind and L. Thorlacius, Nucl. Phys. B382, 123 (1992); T. Banks, A. Dabholkar, M.R. Douglas, and M. O'Loughlin, Phys. Rev. D45, 3607 (1992); T. Banks, M. O'Loughlin and A. Strominger, Phys. Rev. D47, 4476 (1993).

[27] S. Giddings, Phys. Rev. D46, 1347 (1992). 